\title{
Degradation sensitivity of the image quality of UAV remote sensing based on CCD array Motion
}

\author{
Weixin Yang ${ }^{1, a}$, Chuanrong Li ${ }^{1,}{ }^{*}$, Guangyu Liu ${ }^{1}$ \\ ${ }^{1}$ Key Laboratory of Quantitative Remote Sensing Information Technology, Academy of \\ Opto-Electronics, Chinese Academy of Sciences, Beijing 100094,China \\ aemail: wxyang@aoe.ac.cn \\ "Corresponding author, email: crli@aoe.ac.cn
}

Keyword: Sensitivity; Image Quality Evaluation; UAV Remote Sensing

\begin{abstract}
Due to the CCD array and scenery relative motion, the image quality would be degraded. In order to get the relationship between the CCD array move quantity and the image quality, the corresponding degraded images are achieved by changing the CCD array velocity, pitch, roll and yaw variation. Then, the appropriate image quality evaluation method to research the sensitivity of images degraded is required. The results show that the velocity CCD array is the main factor affecting the degradation of the image, and the velocity increase, the image quality degraded sharply, but the motion value has more than 6 pixels in image plane, evaluation values is unchanged basically, the quality of image is extra negative.
\end{abstract}

\section{Introduction}

CCD array has the advantages of short imaging time, high efficiency, and can directly obtain two-dimensional image, it is one of the important loads of remote sensing data. At present, with the continuous improvement of the performance of UAV, it equipped with CCD array to obtain remote sensing image data, so that it is widely used in military [1], resources and environmental science [2], agriculture [3], forestry [4], marine [5], meteorology [6], mapping[7], disaster reduction and disaster prevention[8]. However, in the process of image acquisition, the image quality is degraded by CCD array, which results from the change of the motion state of the UAV platform. This will affect the applications of the image seriously. In order to study the relationship between the CCD array motion state and the image quality, it is very useful to improve the quality of the CCD array image in the future.

The relationship between the moving state of the CCD array and the image quality is studied, including the degradation model, image simulation and image quality evaluation analysis three parts. Liu[9] studied on the effect of image motion on the quality of aerial camera images. Geng[10] analyzed the causes rotation of the image plane and identified by aerial camera like rotational direction method from the image rotation problem. Wang[11] calculated point spread function with the uniform linear motion of payload and simulated the degradations images. Wang[12] focused on inner structure of images and proposed on structural similarity(SSIM) image evaluation method. Li[13] proposed a new frequency domain SSIM image evaluation method. And then, a lot of subjective and objective consistency image evaluation methods[14][15][16] were proposed. However, it is not connected between payload motion and image quality.

In this paper, a new system is researched by using the relationship between CCD array motion and image quality, which informs the sensitivity degradation with CCD array motion values.

\section{CCD array motion degradation model}

CCD array motion degradation of image quality includes four factors, including velocity, pitch, roll and yaw. Image degradation model is illustrated as follows.

(1) CCD array velocity pixel motion values

CCD array by photoelectric stabilized platform mounted on UAV. Compared with UAV velocity, 
the speed change is very tiny. Thus, the CCD array velocity is approximately equal to the UAV speed. The CCD array imaging sketch map of as Fig. 1.

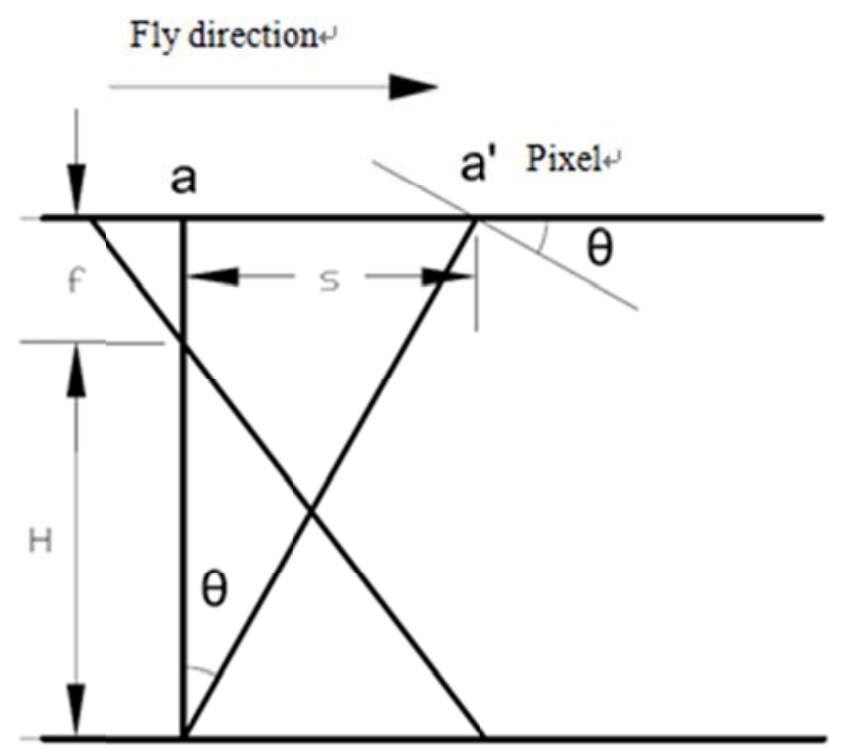

Fig.1. CCD array imaging sketch map

From Fig 1, CCD array motion $\delta_{1}$ by the velocity, it is can be calculate (1).

$$
\delta_{1}=f \frac{V}{H} T
$$

In the type(1): $f$ is focal length of CCD array, $V$ is UAV velocity, $H$ is height between CCD array and ground, $T$ is CCD array imaging time.

(2) CCD array pitch pixel motion values

The pitch of CCD array pixel motion values $\delta_{2}$ can be calculated by (2).

$\delta_{2}=f \cdot \omega_{\theta} \cdot T$

In the type, $\omega_{\theta}$ is the average angular velocity of CCD array pitch motion.

(3) CCD array roll pixel motion values

The roll of CCD array pixel motion values $\delta_{3}$ can be calculated by (3).

$\delta_{3}=f \cdot \omega_{\phi} \cdot T$

In the type, $\omega_{\phi}$ is the average angular velocity of CCD array roll motion.

(4) CCD array yaw pixel motion values

The yaw of CCD array pixel motion values $\delta_{4}$ can be calculated by (4).

$\delta_{4}=L \cdot \omega_{\psi} \cdot T$

In the type (4): $L$ is distance of pixel to rotating center of yaw, $\omega_{\phi}$ is the average angular velocity of CCD array yaw motion.

CCD array pixel motion values are vector, so the motion values by velocity, pitch, roll and yaw, it's meet vector synthesis rule. If synthesis motion values is $\delta$, the corresponding point spread function as follow.

$$
h(x)= \begin{cases}\frac{1}{\delta}, & 0 \leq x \leq \delta-1 \\ 0, & \text { else }\end{cases}
$$

Fourier transformed formula (5), the frequency of PSF as type (6).

$$
H(u)=\frac{\sin (\pi L u)}{\pi L u}
$$




\section{CCD array motion image degradation simulation}

In the imaging time, surface feature and image in CCD array have relative movement, which results in the acquisition of the image degradation. The process of CCD array imaging is showed as Fig.2.

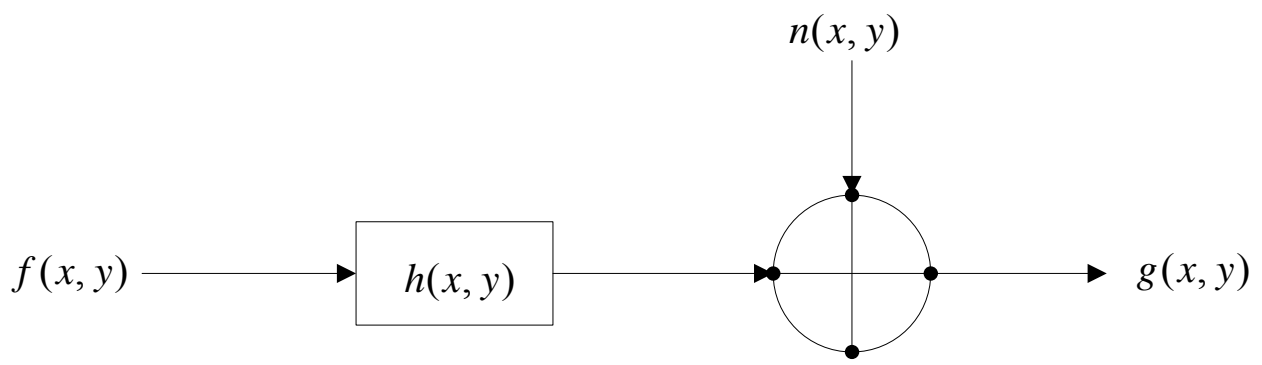

Fig.2. CCD array imaging process

$f(x, y)$ is an ideal image, $h(x, y)$ is PSF by CCD array motion, $n(x, y)$ is an noise, $g(x, y)$ is actual image. This process can be described by type (7).

$$
g(x, y)=f(x, y) * h(x, y)+n(x, y)
$$

In order to simulate degradation image, choosing parameters are demonstrated. The maximum height of UAV flight is $5000 \mathrm{~m}$, the fastest speed is $180 \mathrm{~m} / \mathrm{s}$. The CCD array imaging time is $1 \mathrm{~ms}$, focus length $0.65 \mathrm{~nm}$, pixel size $6.5 \times 10^{-6} \mathrm{~m}$. pitch roll and yaw angular speed $0.6^{\circ} / \mathrm{s}$, Maximum rotation radius of the focal plane $10.7464 \mathrm{~mm}$.

After Calculating, it is illustrated that the CCD array pitch, roll and yaw image shift amount is far less than the speed generated image shift amount. Therefore, study CCD array image degradation by motion sensitivity problem can be approximately equivalent to the speed of image degradation caused sensitivity analysis of research questions.

According to type (1), the motion values by the CCD array velocity can be calculated, the results show in table.1.

Table.1. CCD array velocity motion pixel values

\begin{tabular}{|c|c|c|c|c|c|c|}
\hline $\mathrm{V} / \mathrm{H}^{-1} \mathrm{~s}^{-1}$ & 0.1 & 0.2 & 0.3 & 0.4 & 0.5 & 0.6 \\
\hline$\delta_{1}$ & 1.0231 & 2.0462 & 3.0692 & 4.0923 & 5.1154 & 6.1385 \\
\hline $\mathrm{V} / \mathrm{H}^{-1} \mathrm{~s}^{-1}$ & 0.7 & 0.8 & 0.9 & 1.0 & 1.1 & 1.2 \\
\hline$\delta_{1}$ & 7.1615 & 8.1848 & 9.2077 & 10.2308 & 11.2538 & 12.2769 \\
\hline $\mathrm{V} / \mathrm{H}\left(\mathrm{s}^{-1}\right)$ & 1.3 & 1.4 & 1.5 & 1.6 & 1.7 & 1.8 \\
\hline$\delta_{1}$ & 13.3 & 14.3231 & 15.3462 & 16.3692 & 17.3923 & 18.4154 \\
\hline
\end{tabular}

Thus, simulating degraded image when motion values are five pixels. The original image and degraded image are showed as follow. 


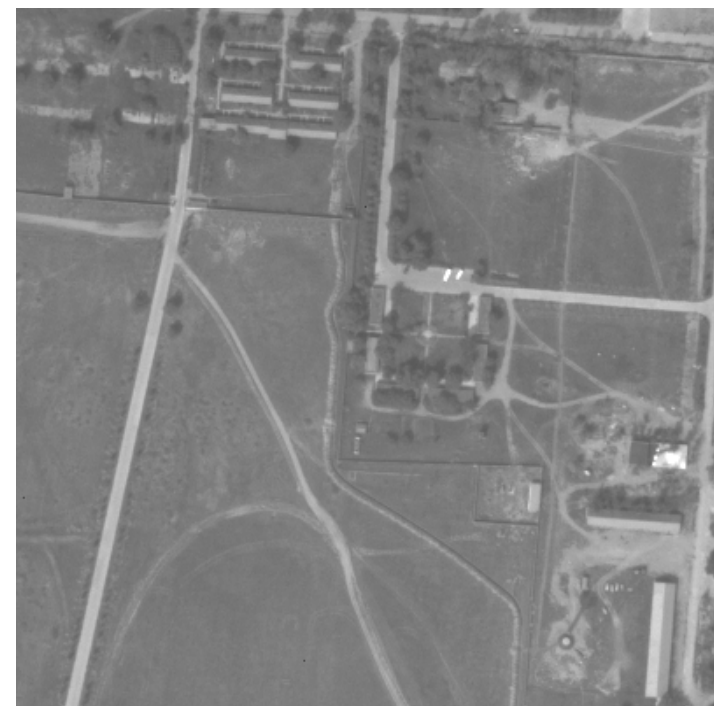

(a) Original image

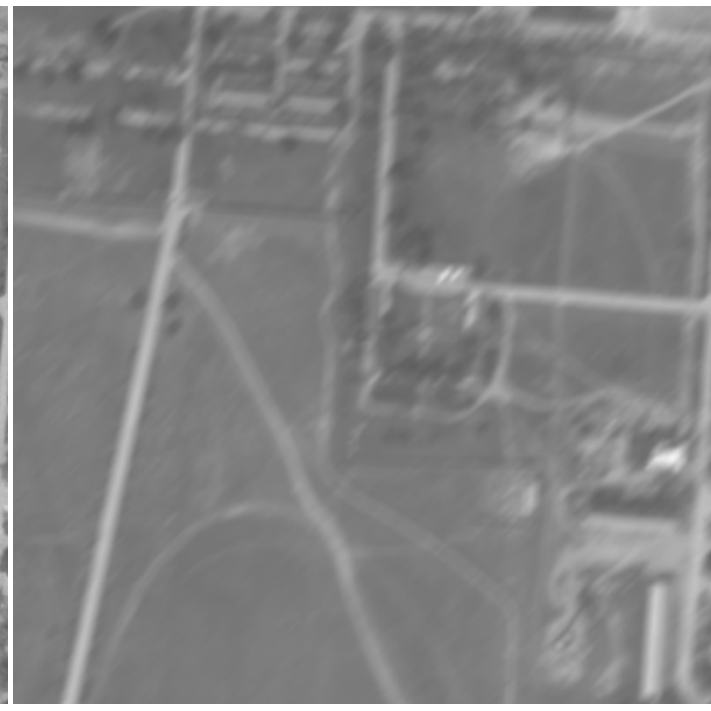

(b) Degraded image size is 5 pixels

\section{CCD array image degradation motion sensitivity analysis}

The choosing appropriate image quality evaluation method is so useful to study the sensitivity of image degradation. The evaluation of subjective and objective consistency, the choose of gradient definition (GD)[12], Relative Edge Response (RER) are required to evaluated degraded images. The results from evaluated degradation are showed as table. 2.

Table.2. image quality evaluation results

\begin{tabular}{|c|c|c|c|c|c|c|c|}
\hline $\mathrm{V} / \mathrm{H}\left(\mathrm{s}^{-1}\right)$ & $\delta_{1}$ & GD & RER & $\mathrm{V} / \mathrm{H}\left(\mathrm{s}^{-1}\right)$ & $\delta_{1}$ & GD & RER \\
\hline 0.1 & 1.0231 & 0.307 & 0.46 & 1.0 & 10.2308 & 0.019 & 0.2 \\
\hline 0.2 & 2.0462 & 0.206 & 0.34 & 1.1 & 11.2538 & 0.018 & 0.2 \\
\hline 0.3 & 3.0692 & 0.181 & 0.31 & 1.2 & 12.2769 & 0.0177 & 0.2 \\
\hline 0.4 & 4.0923 & 0.083 & 0.23 & 1.3 & 13.3 & 0.0173 & 0.21 \\
\hline 0.5 & 5.1154 & 0.063 & 0.22 & 1.4 & 14.3231 & 0.0172 & 0.21 \\
\hline 0.6 & 6.1385 & 0.03 & 0.19 & 1.5 & 15.3462 & 0.0172 & 0.22 \\
\hline 0.7 & 7.1615 & 0.024 & 0.2 & 1.6 & 16.3692 & 0.0172 & 0.18 \\
\hline 0.8 & 8.1848 & 0.022 & 0.19 & 1.7 & 17.3923 & 0.0173 & 0.21 \\
\hline 0.9 & 9.2077 & 0.02 & 0.21 & 1.8 & 18.4154 & 0.0168 & 0.17 \\
\hline
\end{tabular}

Draw a graph showing changes in each of the evaluation results, It can directly reflect the image quality degradation. The graphs show Fig 4 to 5 . 


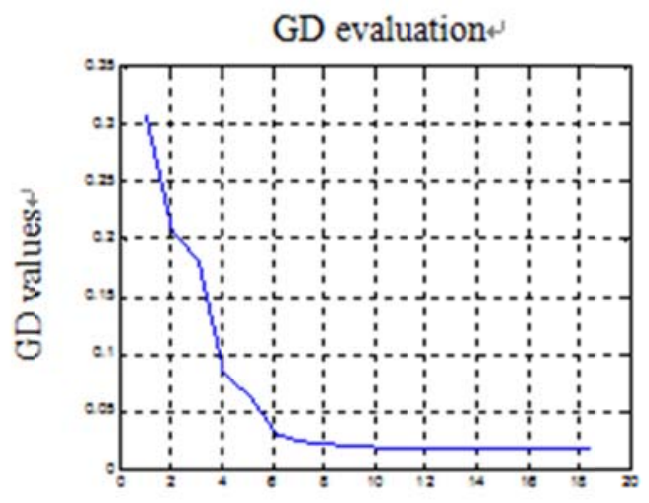

Velocity pixel motion values

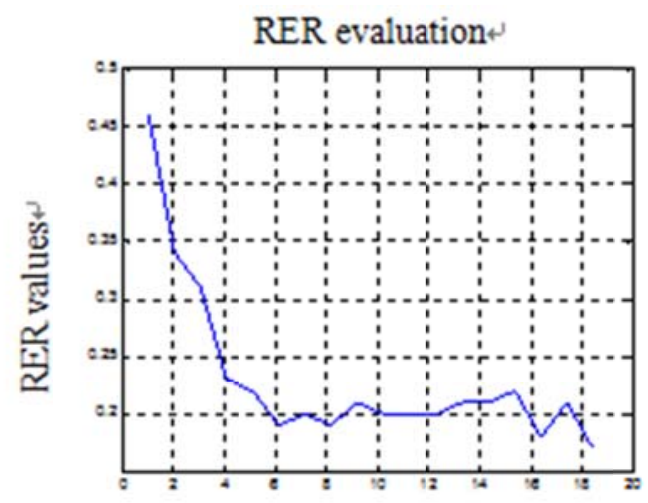

Velocity pixel motion values

Fig.4. GD evaluation

Fig.5. RER evaluation

The conclusion of the consistency results from the evaluations. (1) following the increase in the velocity height ratio, and pixel motion values are rising, the image becomes deeply blur, GD and RER values are declining . (2) before pixel motion values less than 6, GD and RER values decrease sharply, it is show that the quality of image degradation is clear. However, the motion value more than 6 pixels in image plane, evaluation values is basically unchanged, which has negative effects on the image quality.

The results leads to the fact that following the forward velocity of CCD array occurs in the imaging process, the feature image in the focal plane relative displacement occurs. The motion values and the blurred image result in GD and RER values reduction. When the motion values increase to the threshold, the image quality is deeply blurred, the evaluation GD and RER values are extra small and basically unchanged.

\section{Conclusion}

In this paper, by simulating the images degradation and evaluation, the process of the CCD array image analysis illustrates the effect of image quality by payload movement. It indicates that when the motion values achieve 6 pixels, the quality of image is extra negative. However, the research does not consider the effect of back attitude change of CCD array image quality. Therefore, the image quality degradation takes account in all factors and gets a full sensitivity by motion parameters in the further study.

\section{Acknowledgement}

In this paper, the research was sponsored by the National High-tech R\&D Program (863 Program No. 2013AA122102).

\section{References}

[1] Samad T, Bay J S, Godbole D. Network-centric systems for military operations in urban terrain: the role of UAVs[J]. Proceedings of the IEEE, 2007, 95(1): 92-107.

[2] Ruisheng Ma, Han Sun, Lunji Ma, et al. Land-use Survey Based on Image from Miniature Unmanned Aerial Vehicle [J]. Remote Sensing Information, 2006.

[3] Grenzdörffer G J, Engel A, Teichert B. The photogrammetric potential of low-cost UAVs in forestry and agriculture[J]. The International Archives of the Photogrammetry, Remote Sensing and Spatial Information Sciences, 2008, 31(B3): 1207-1214.

[4] Merino L, Caballero F, Martínez - de Dios J R, et al. A cooperative perception system for multiple UAVs: Application to automatic detection of forest fires[J]. Journal of Field Robotics, 2006, 23(3-4): 165-184. 
[5] Lomax A S, Corso W, Etro J F. Employing unmanned aerial vehicles (UAVs) as an element of the integrated ocean observing system[C]//OCEANS, 2005. Proceedings of MTS/IEEE. IEEE, 2005: 184-190.

[6] Roth A M, Helmke M F. High times in the Great Valley: Remote sensing by unoccupied aerial vehicle (UAV)[C]//Geological Society of America Abstracts with Programs,. 2006, 38(2): 25.

[7] Harwin S, Lucieer A. Assessing the Accuracy of Georeferenced Point Clouds Produced via Multi-View Stereopsis from Unmanned Aerial Vehicle (UAV) Imagery [J]. Remote Sensing, 2012, 4(6): 1573-1599.

[8] James, M. R, Niethammer, U, Traveletti, J, Topographic reconstruction of landslide surfaces using images from an unmanned aerial vehicle. In: ISPRS Commission V Mid Term Symposium, Newcastle, UK. 2010.

[9] Ming Liu, Gang Liu, Youyi Li, Haipeng Kuang, Jihong Xiu, Linpei Zhai. The effect of image motion on the quality of aerial camera images[J]. Optics and Precision Enginering, 2004,(31):12-14.

[10] Wenbao Geng, Lin-pei Zhai, YaLin Ding, Xiuying Zhao. Characteristic anakysis for image rotation of aerial camera [J]. Infrared and Laser Engineering. 2008,37 (6):1053-1057.

[11] Wang Z, BOVIK A C, SHEIKH H R, et al. Image Quality Assessment: From Error Visibility to Structural Similarity [J]. IEEE Transactions on Image Processing, 004,13(4):600-612.

[12] Xiaohong Wang, Rongchun Zhao. Estimating the PSF of Motion-blurred images [J]. 2001.21(9):40-41.

[13] Hang Li, Yang Lu, HuiJuan Cui, Kun Tang. Image quality assessment based on frequency domain based structural similarities [J]. Tsinghua Univ(Sci\&Tech), 2009, 4(49):559-562.

[14] Tianpeng Xie, Miaozhong Xu, Ming Cong, Xiaoling Zhu. Research on No-reference Remote Sensing Image Quality Assessment Based on Contourlet -GSSIM [J]. Bulletin of Surveying and Mapping. 2015(1):59-62.

[15] Xiaoling Zhu, Miaozhong $\mathrm{Xu}$, Ming Cong. Assessment Method for No-reference Remote Sensing Image Quality Based on Support Vector Machine [J]. Spacecraft recovery \& Remote Sensing. 2014.12(35):83-90.

[16] Yang Diwei. Research on Methods Image Quality Assessment Based on Natural Scene Statistics [D]. China University of Geosciences Wuhan. 2014. 